\title{
Über die Einwirkung von Jodmethyl und Kali auf Phloroglucin
}

\author{
von \\ Otto Margulies. \\ Ars dem chemischen Laboratorium des Prof. Ad. Lieben \\ an der k. k. Universität in Wien.
}

(Vorgelegt in der Sitzung am 25. October 1888.)

Im Einverständniss mit den Herren Dr. Herzig und Zeisel und im Anschluss an deren unter dem Titel: „Neue Beobachtungen über Bindungswechsel bei Phenolen" ${ }_{1}$ veröffentlichte Arbeit, habe ich die Einwirkung von Jodmethyl und Kali auf Phloroglucin studiert.

Ich begann diese Untersuchung in der Erwartung, dass entweder die Reaction im Ganzen und Grossen so verlaufen werde, wie die von Herzig und Zeisel studierte, und möglicherweise gerade jene Methylsubstitutionsproducte des Phloroglucins, die den nicht krystallisirten Äthylphloroglucinen dieser Forscher entsprechen, in fassbarer Form erhalten werden könnten, oder dass die Producte der Methylirung des Phloroglucins vielleicht einem ganz anderen Typus, sei es dem des secundären oder bitertiären Phloroglucins angehören könnten.

Das Experiment hat nun gezeigt, das neben Homologen des bisecundären Phloroglucins, und zwar als Hauptproduct, pentamethylirtes secundäres Phloroglucin entsteht, und darin unterscheidet sich der Verlauf der Einwirkung von Jodmethyl und Kali auf Phloroglucin wesentlich von dem von Herzig and Zeisel studierten Processe.

1 Monatshefte f. Chemie, IX., S. 217 u. 882.

Chemie-Heft Nr. 10. 


\section{Methylirung von Phloroglucin mit Jodmethyl und Kali.}

Dem Verhältniss von 1 Molekual Phloroglucin zu 6 Molekülen Ätzkali entsprechend, trug ich in eine 10percentige, lanwarme Aufösung von Kali in 97percentigem Alkohol, nach dem Verfahren von Will gereinigtes Phloroglucin ein und liess Jodmethyl zufliessen. Die Reaction verlief anfangs unter freiwilliger Erwärmung und wurde auf dem kochenden Wasserbade am Rückflusskühler zu Ende gefübrt. Der Alkohol wurde abdestillirt und der Rückstand in Wasser aufgenommen, wobei sich ein Öl abschied. Behufs Trennung der in Kali löslichen Producte von in Kali unlöslichen, wurde der Kolbeninhalt mit überschüssigem Kali versetzt, mit $\ddot{A}$ ther ausgeschiittelt und der Äther so lange mit wässeriger Kalilauge gewaschen, bis die Waschlange farblos ablief. Die zuerst erhaltene, alkalische Flüssigkeit, mit den Waschlaugen vereinigt, wurde angesäuert, mit Äther ausgeschiittelt und dieser hinterliess nach dem Abdestilliren ein harziges, braungefärbtes Product, das wieder in derselben Weise wie oben mit Jodmethyl, Kali und Alkobol behandelt wurde, und wieder in Kali lösliches und in Kali unlösliches Product in ätherischem Auszuge lieferte, welch' letzteres mit dem bei der ersten Operation erhaltenen vereinigt wurde.

\section{Die in Kali unlöslichen methylirten Phloroglucine}

haben sich als ein Gemenge von secundärem Pentamethylphloroglucin mit den Monomethyläthern des dreifach und vierfach methylirten secundären Phloroglucins erwiesen. Die ätherischen Lösungen des in Kali unlöslichen Productes hinterliessen nach dem Abdestilliren des Äthers einen Riickstand, der aber nicht immer dieselbe Beschaffenheit und Zusammensetzung besass. Bei den ersten Versuchen erhielt ich diese Snbstanz als dunkel gefärbtes, nicht krystallisirendes öl. Später, als ich von einem reineren Phlorogluein ausging, erhielt ich sie in Form eines hellgelb gefärbten Liquidum's, das nach einigem Stehen zu Decimeter langen, spiessigen Krystallen, die von einem öl durchtränkt waren, erstarrte. 
Die Analysen, die an dem Rohproducte verschiedener Darstellungen vorgenommen wurden, zeigten, dass auch seine $\mathrm{Zu}$ sammensetzung eine nicht constante war. Die Zahlen bewegten sich innerhalb der für Tetra- und Pentamethylphloroglnein berechneten Werthe. Dieser in Kali unlösliche Theil der Reactionsproducte war daher unzweifelhaft ein von Fall zu Fall in seiner Zusammensetzung wechselndes Gemenge.

Methoxylbestimmungen, die mit dem Rohproducte ausgeführt wurden, zeigten, dass wenigstens ein Theil der eingetretenen Methylgruppen an Sauerstoff gebunden war.

Der Hauptsache nach aber besteht dieses Gemenge aus

\section{Pentamethylphloroglucin,}

eben jenem schön krystallisirenden Körper, der vorhin flüchtig erwähnt wurde. Einmal im Besitz von Krystallen dieser Verbindung, war es mir ein Leichtes, durch Aussaat derselben das in Kali unlösliche Rohproduct jedesmal zum theilweisen Erstarren zu bringen.

Von den Krystallen wurde abgesaugt und dieselben mehrmals aus Aether umkrystallisirt, bis die Substanz den constanten und scharfen Schmelzpunkt $80^{\circ} \mathrm{C}$. zeigte.

Nachfolgende Analysen wurden an Producten verschiedener Darstellungen ausgeführt:

I. $0 \cdot 2388 g$ Substanz gaben $0 \cdot 5897 g$ Kohlensäure u. $0 \cdot 1765$ Wasser

II. $0.2454 " n \quad 0 \cdot 6074 \quad n \quad 0 \cdot 1845 n$

In 100 Theilen:

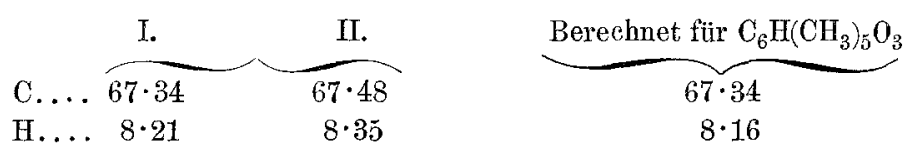

Als ich in der Erwartung, dass hier der Methyläther des bisecundären Tetramethylphloroglucins vorliege, versuchte, den Methoxylgehalt der Substanz zu bestimmen, nabm ich zu meiner Überraschung wahr, dass beim Kochen mit Jodwasserstoff auch nicht die Spur Jodmethyl gebildet wurde, denn anch nach zwei- 
stündigem Kochen mit Jodwasserstoff im Zeis e l'schen Apparate wurde kein Jodsilber ausgeschieden.

Die Verbindung enthält demnach keine der fünf eingetretenen Methylgruppen an Sauerstoff gebunden. Sie enthält ausserdem kein Hydroxyl, denn sie ist in Kali unlöslich, und überdies konnte festgestellt werden, dass eine alkoholische Lösung derselben auf Zusatz auch einer sehr geringen Menge Bromwasser bleibend gelb gefärbt wurde, dass somit die Substanz nicht fähig ist, Brom additionell aufzunehmen.

Die Annahme von doppelt gebundenen Kohlenstoffatomen in diesem Pentamethylphloroglucin ist daher nicht statthaft. Damit harmonirt auch das Verhalten der Verbindung gegen Oxydationsmittel. ${ }^{1}$ Von Kaliumpermanganat wird das in Essigsäure gelöste Pentamethylploroglucin selbst nach mehrstïndigem Erhitzen nicht angegriffen. Ebenso resistent verhielt sich die Substanz gegen concentrirte Salpetersäure selbst in der Kochhitze.

Alle diese Beobachtungen zusammengenommen lassen fur das Pentamethylphloroglucin kaum eine andere Formel als die eines pentamethylirten secundären Phloroglucins als möglich erscheinen:<smiles>CC(C)(C)C(=O)C(C)(C)C(=O)C(C)(C)C</smiles>

Bloss eine Beobachtung steht mit einer solchen Deutung der Constitution des Körpers nicht in gutem Einklange: die Indifferenz

1 Siehe Baeyer Ann. d. chem. 245. S. 247.

Ich habe zwar nicht, wie Baeyer, alkalisches Kaliumpermangan at als Oxydationsmittel in Anwendung gebracht, glanbe aber, und es liegen dafür viele Beispiele vor, dass ungesättigte Verbindungen auch von sauern Oxydationsmitteln angegriffen werden müissten. 
des Pentamethylphloroglucins gegen Phenylhydrazin. In alkoholischer Lösung. mit diesem Reagens erhitzt, blieb die Verbindung ganz unverändert.

Die Einwirkung von Hydroxylamin wurde bis jetzt nicht versucht.

Da das in Kali unlösliche Rohproduct, wie oben nachgewiesen, methoxylbältige Verbindungen enthielt, das Pentamethylphloroglucin aber methoxylfrei ist, so mussten sich in den öligen Mutterlaugen des letzteren jene Körper vorfinden. In der That konnte auch nachgewiesen werden, dass neben gelöstem Pentamethylphlorogluein die Methyläther des Tri- und Tetramethylphloroglucins darin vorhanden sind.

Das ölige Filtrat des Pentamethylphloroglucins wurde der Destillation im Vacnum unterworfen, und es ging hiebei die Hauptmenge des Öles bei $30 \mathrm{~mm}$ Druck zwischen $153-155^{\circ} \mathrm{C}$ anscheinend unzersetzt über.

Eine vollständigere Trennung der Gemengtheile wurde jedoch erst durch Abkülllen des Destillates, welches neue Krystallansätze zeigte, erzielt, indem die ausgeschiedenen Krystalle ron noch vorhandenem Öle bei niedriger Temperatur durch Absangen and Abpressen getrennt wurden.

Die Krystalle erwiesen sich als Pentamethylphloroglucin.

Der auch in der Kälte ölig verbliebene Antheil wurde mit Jodwasserstoff gekocht und die gebildeten Producte mit Äther extrahirt.

Die Ätherlösungen wurden, um secundäres Pentamethylphloroglucin von in Kali löslichen Producten zu trennen, mit Kalilange behandelt, die kalischen Lösungen vereinigt, angesäuert und mit Äther extrabirt. Der Äther hinterliess nun einen theilweise krystallinischen Rückstand, der sich als aus zwei verschiedenen Verbindungen bestehend erwies. Es blieb nämlich ein Theil davon selbst beim Kochen mit Benzol ungelöst, von dem abfiltrirt wurde.

Der ungelöst gebliebene Antheil wurde aus sehr verdünntem Methylalkohol unkrystallisirt, und endlich in Form von farblosen, dünnen, rechtwinklig begrenzten Täfelchen mit dem Schmelzpunkt $184^{\circ} \mathrm{C}$. erhalten. 
Die Verbrennung ergab folgendes auf

\section{Trimethylphloroglucin}

stimmendes Resultat:

$0 \cdot 2144 g$ Substanz gaben $0 \cdot 5071 g$ Kohlensäure und $0 \cdot 1380$ Wasser

In 100 Theilen:

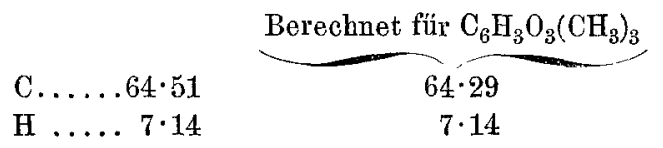

Das Trimethylphloroglucin ist fähig, ein Wasserstoffatom gegen Metalle auszutauschen. So ist es in Kalilange und Natriumcarbonat leicht löslich. Eine acidimetrische Bestimmung zeigte dass 1 Molekül der Verbindung durch 1 Molekül Ätznatron neutralisirt wird.

$0 \cdot 1016 \mathrm{~g}$ Substanz sättigten $1.8 \mathrm{~cm}^{3}$ einer Natronlauge vom Titre

$0 \cdot 01355 \mathrm{~g} \mathrm{NaOH}$, während die Bildung von $\mathrm{C}_{9} \mathrm{H}_{11} \mathrm{Na}_{3} 1.78 \mathrm{~cm}^{3} \mathrm{NaOH}$ erfordert.

Die alkoholische Lösung des Trimethylphloroglucins nahm schon in der Kälte Brom unter Entfärbung auf. Nachdem soviel Brom hinzugefügt war, dass die Flüssigkeit danernd gelb gefärbt. blieb, wurde durch Zusatz von Wasser ein krystallinischer, bromhältiger Körper gefällt. Im Filtrat war Bromwasserstoffsäure deutlich nachweisbar. Leider gestattete mir die kleine Menge Substanz, die für diesen Versuch verfügbar war, nicht eine exacte quantitative Bestimmung der verbrauchten Menge Brom und der gebildeten Menge Bromwasserstoff.

Das krystallinische Bromproduct löste sich leicht in Kali, liess sich aber durch Säure schwer wieder ausfällen. Sein Schmelzpunkt war $90^{\circ} \mathrm{C}$.

Auf Zusatz von Kaliumpermanganat zur Natriumcarbonatlösung des Trimethylphloroglucins trat augenblickliche Reduction des Permanganates ein.

Man konnte demnach das Trimethylphloroglucin für eine ungesättigte Verbindung halten, wenn auch der Verlauf der Einwirkung von Brom sich nicht als ein glatter Additionsprocess 
erwiesen hatte. Eine einfache additionelle Vebindung des Trimethylphloroglucins mit Halogenen ist aber auch von vorne herein nicht zu erwarten, denn welche von den fünf nachfolgenden Formeln wir dem Trimethylphloroglucin auch zuschreiben,
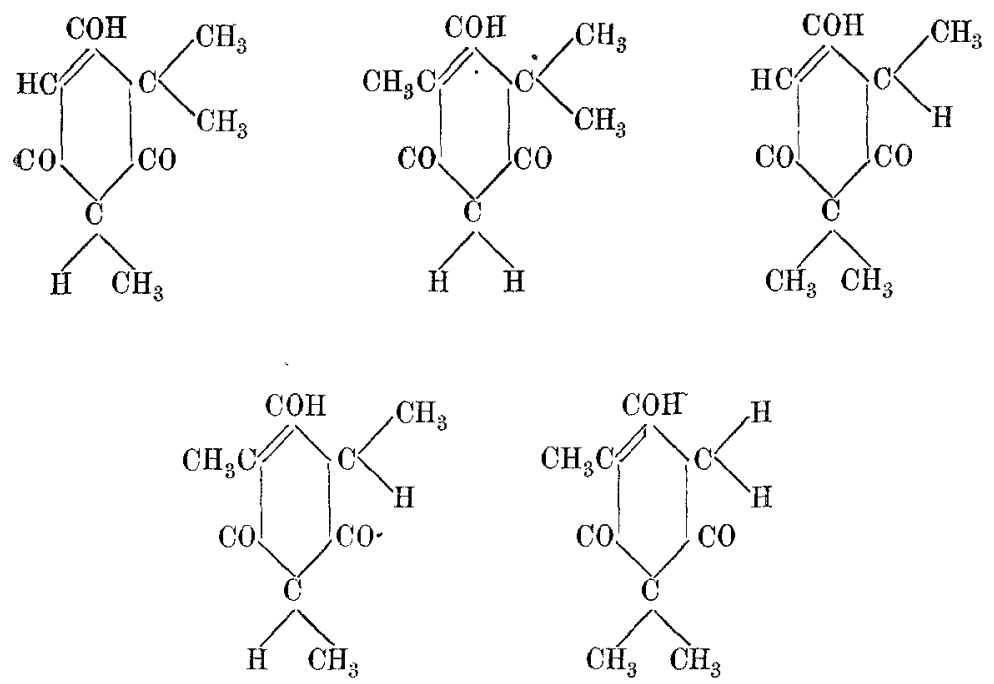

so wird doch der erste Effect der Bromaddition die Bildung einer unbeständigen Gruppe $\mathrm{C}(\mathrm{OH})$ Br sein, die sich unter $\mathrm{Ab}$ spaltung von $\mathrm{HBr}$ in $\mathrm{CO}$ umwandeln muss. Es wäre dadurch erklärt, warum Bromwasserstoff gebildet wird, obwohl die äusseren Erscheinungen der Einwirkung von Brom den gewöhnlich bei einer Addition beobachteten entsprechen.

Eine weitergehende Discussion an das Verhalten des Trimethylphloroglucins gegen Brom zu knüpfen, halte ich für verfrüht, so lange dieser Vorgang nicht einem genaueren Studium unterworfen ist.

\section{Bisecundäres Tetramethylphloroglucin.}

Ausser dem Trimethylphloroglucin liess sich aus den mit Jodwasserstoffsäure gekochten öligen Mutterlaugen des Pentamethylphloroglucins noch eine zweite Verbindung gewinnen, die 
sich vermöge ihrer Löslichkeit in Benzol leicht vom Trimethylphloroglucin trennen liess.

Ich erhielt den Körper in Form von farblosen, kleinen Nadeln, die den Schmelzpunkt $114^{\circ}$ C. zeigten.

Die Verbrennung stimmte anf Tetramethylphloroglucin:

$0 \cdot 1512 g$ Substanz gaben $0 \cdot 3638 g$ Kohlensäure und $0 \cdot 1066 g$ Wasser

In 100 Theilen:

$$
\begin{aligned}
& \text { C.....65.61 } \\
& \text { H..... } 7 \cdot 80
\end{aligned}
$$

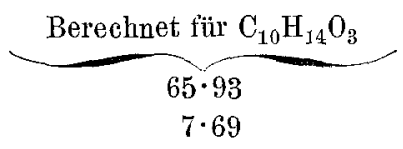

Der Körper ist in Natrinmearbonat leicht löslich, und auf" Zusatz von Kaliumpermanganat trat sofort Reduction ein.

Auch hier wurde eine acidimetrische Bestimmung vorgenommen, und es zeigte sich, dass 1 Molekül der Verbindung. durch 1 Moleküil Ätznatron nentralisirt wird.

$0 \cdot 3410 \mathrm{~g}$ Substanz sättigten $5 \cdot 4 \mathrm{~cm}^{3}$ Natronlauge von Titre $0.01355 \mathrm{~g} \mathrm{NaOH}$ während die Bildung von $\mathrm{C}_{10} \mathrm{H}_{13} \mathrm{O}_{3} \mathrm{Na} 5.54 \mathrm{~cm}^{3}$ erfordert.

Auch eine quantitative Bromaddition wurde ansgeführt, aus. der hervorging, dass die Verbindung zwei Atome Brom addirt, die Hälfte des addirten Broms aber auf Wasserzusatz als Bromwasserstoff abspaltet.

Es wurden $0 \cdot 2797 \mathrm{~g}$ Substanz in kalt gehaltener alkoholischer Lösung bis zur bleibenden Gelbfärbung mit Brom versetzt. Es wurden $0.2308 \mathrm{~g}$ Brom verbraucht, während dem Verhältniss $\mathrm{C}_{10} \mathrm{H}_{14} \mathrm{O}_{3}: \mathrm{Br}_{2} 0.2453 \mathrm{~g}$ Brom entsprechen. Auf Wasserzusatz fiel ein krystallinisches Bromproduct aus, das zwischen $64^{\circ}$ und $68^{\circ} \mathrm{C}$. schmolz. Im Filtrat wurden $0.1322 \mathrm{~g}$ als Bromwasserstoff vorhandenes Brom, d. i. $57 \cdot 4 \%$ des ursprïglich zugesetzten. gefunden.

Man darf nach dem Vorausgegangenen annehmen, dass das Tetramethylphloroglucin eine Hydroxylgruppe enthält, die ihm Phenolcharakter verleiht. Mit Rücksicht auf seine genetische Beziehung zum Phloroglucin wird für diese Verbindung eine der drei folgenden Formeln in Anspruch genommen werden müssen: 
Phlorogluein.
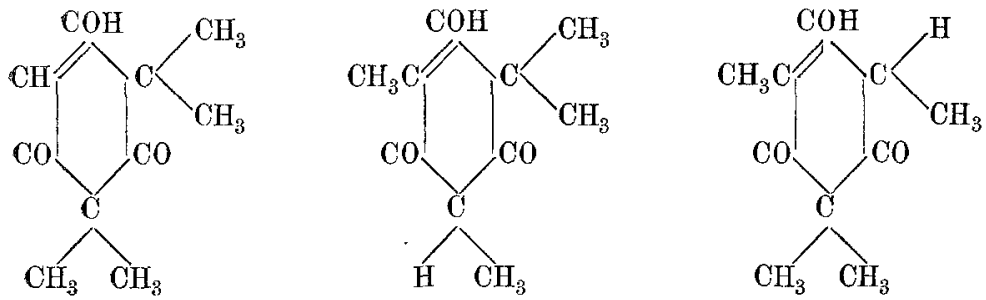

Jede der drei Formeln lässt erwarten, dass die Verbindung im Stande sein könnte, zwei Atome Brom additionell aufzulnehmen, und die Hälfte des addirten Broms in Folge der intermediären Bildung der unbeständigen Gruppe $\mathrm{C}(\mathrm{OH}) \mathrm{Br}$ als Bromwasserstoff abzuspalten. Die vorhin für diese Reaction gegebenen Zahlen stehen mit dieser Interpretation in ziemlich gutem Einklange. Immerhin ist noch eine eingehendere Untersuchung des Bromirungsproductes des Tetramethylphloroglucins sehr wiinschenswerth.

\section{In Kali lösliche Phloroglucine.}

Die bei der Methylirung des Phlorogltucins erhaltene alkalische Flïssigkeit enthält neben braunen, nicht krystallisirenden Körpern, über deren Natur mir bis jetzt nichts bekannt ist, ebendasselbe Tri- und Tetramethylphloroglucin, deren Darstellung aus dem in Kali unlöslichen öle ich soeben beschrieben habe, und die im ursprïnglichen Reactionsgemenge wohl nur als Monomethyläther enthalten gewesen sein konnten.

Aus dem rohen Gemisch dieser beiden Phloroglucine, wie es als in Kali lösliches Reactionsproduct erhalten worden war, wurden beide Substanzen isolirt, wie oben rein dargestellt, und sie zeigten die oben angegebenen Schmelzpunkte $184^{\circ}$ und $114^{\circ} \mathrm{C}$.

Die Verbrennungen ergaben:

$$
\text { Trimetbylphlorogluein }
$$

0. $1832 g$ Substanz gaben $0.4337 g$ Kohlensäure und $0.1188 g$ Wasser.

In 100 Theilen:
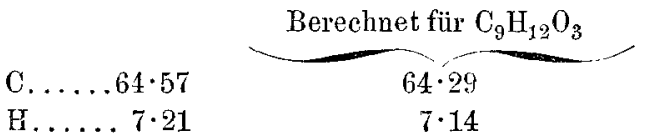
$0.2180 \mathrm{~g}$ Substanz gaben $0.5254 \mathrm{~g}$ Kohlensäure und $0.1566 \mathrm{~g}$ Wasser In 100 Theilen :

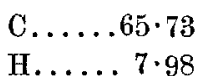

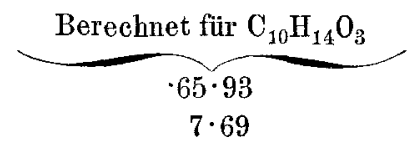

Ich konnte auch feststellen, dass beide Substanzen, zwei Stunden im Zeisel'schen Apparate mit Jodwasserstoffsäure gekocht, keine Abscheidung ron Jodsilber hervorriefen, und die aus der Jodwasserstoffsäure zurïckgewonnenen Verbindungen crwiesen sich als unverändert.

Die Identität dieser aus dem Reactionsproducte der Phloroglucinmethylirung direct erhaltenen Verbindungen mit dem früher beschriebenen aus seinem Monometbyläther gewonnenen Trirespective Tetramethylphlorog]ucin, erscheint daher genügend erwiesen. 